\title{
Spectroscopy of high proper motion stars in the ground-based UV
}

\author{
V. Klochkova ${ }^{*}$, T. Mishenina ${ }^{2}$, S. Korotin ${ }^{2}$, V. Marsakov $^{3}$, \\ V. Panchuk ${ }^{1}$, N. Tavolganskaya ${ }^{1}$ I. Usenko ${ }^{2}$ \\ * valenta@sao.ru
}

\begin{abstract}
Based on high quality spectral data (spectral resolution $\mathrm{R} \geq 60000$ ) within the wavelength range of $3550-5000 \AA$ we determined main parameters (effective temperature, surface gravity, microturbulent velocity, and chemical element abundances including heavy metals from $\mathrm{Sr}$ to Dy) for 14 metal-deficient $\mathrm{G}-\mathrm{K}$ stars with large proper motions. The stars we studied have a wide range of metallicity: $[\mathrm{Fe} / \mathrm{H}]=-0.3 \div-2.9$. Abundances of $\mathrm{Mg}, \mathrm{Al}, \mathrm{Sr}$ and Ba were calculated with non-LTE line-formation effects accounted for. Abundances both of the radioactive element Th and $\mathrm{r}$-process element Eu were determined using synthetic spectrum calculations. We selected stars that belong to different galactic populations according to the kinematical criterion and parameters determined by us. We found that the studied stars with large proper motions refer to different components of the Galaxy: thin, thick disks and halo. The chemical composition of the star $\mathrm{BD}+80^{\circ} 245$ located far from the galactic plane agrees with its belonging to the accreted halo. For the giant HD 115444 we obtained $[\mathrm{Fe} / \mathrm{H}]=-2.91$, underabundance of Mn, overabundance of heavy metals from Ba to Dy, and, especially high excess of the $\mathrm{r}-$ process element Europium: $[\mathrm{Eu} / \mathrm{Fe}]=+1.26$. Contrary to its chemical composition typical for halo stars, HD 115444 belongs to the disc population according to its kinematic parameters.
\end{abstract}

Subject headings: high resolution spectroscopy - ground-based UV and blue region metal-poor stars - chemical composition

\section{Introduction}

Spectral and kinematical parameters of unevolved stars that belong to different galactic populations are the main sources of information about chemical and kinematical evolution of the Galaxy.

\footnotetext{
${ }^{1}$ Special Astrophysical Observatory, Nizhnij Arkhyz, Russia

${ }^{2}$ Odessa Astronomical Observatory, Odessa, Ukraine

${ }^{3}$ South Federal University, Rostov-Don, Russia
} 
We see that spectroscopy of stars of different generations in the Galaxy is a key program for the largest telescopes in the world. During last decades scientists become more and more interested in spectroscopic studies of the oldest stellar populations, since chemical evolution of the Galaxy is imprinted in the chemical composition of metal-poor stars of subsequent generations. To reconstruct the chronology of the chemical and kinematic evolution of the Galaxy we need an enormous number of high quality spectral data and accurate proper motion and parallax determination for metal-deficient stars.

Unevolved F-G stars (dwarfs and subdwarfs) are the most effective probes of the chemical composition and measurements of radial velocities. Their spectra are abundant in narrow and low-blended absorptions as seen at a high spectral resolution. In order to enlarge the sample of unblended lines, we have to record a broad spectral region. For extremly metal-deficient stars that have weak lines, the spectra have to be taken in the blue and ultraviolet regions where there are more lines than in the visible range. Considering the facts mentioned above, we performed high resolution spectroscopy of a sample of $\mathrm{F}-\mathrm{G}$ metal-poor stars at the 6-meter telescope of the Special Astrophysical Observatory of the Russian Academy of Sciences. All our spectra were taken within a broad spectral interval from 3550 to $5000 \AA$ A. Below we present some recent results.

\section{Spectral observations and spectra reduction}

We have selected a sample of high-velocity stars from the high proper motion stars review (Carney et al. 1994). HD/BD numbers of 14 stars are listed in Table1. The spectral data were taken using the échelle spectrograph NES (Panchuk et al. 2009) permanently mounted in a Nasmyth focus of the 6-meter telescope of the Special Astrophysical Observatory. NES provides a spectral resolving power of $\mathrm{R} \geq 60000$ in the spectral range $3000-10000 \AA$. NES is capable to observe in the UV due to the camera made of fused silica. Now NES works in combination with a $2048 \times 2048$ pixel CCD that has a high sensitivity in the ground-based UV spectral range, for wavelengths longer of $3000 \AA$. We notice that spectral range of the spectrograph NES is being crossed with the range (1740-3100 $)$ of the high resolution spectrograph UVES (Kappelmann et al. 2006) which is projected for the World Space Observatory-UV (more details see in the paper by Shustov et al. 2009).

The 2D échelle-spectra were reduced (applying standard procedures of bias subtraction, scattered light and cosmic ray trace removal, and order extraction) using the context ECHELLE of the system MIDAS. The context ECHELLE was modernized to process spectra obtained in combination with an image slicer (Yushkin \& Klochkova 2004). The signal-to-noise ratio for all the spectra discussed in this paper is higher than 200. Combined with the spectral resolving power, that allowed us not only to detect rather weak lines but also to study their profiles.

Kinematic characteristics (proper motions and parallaxes) of the stars we study are presented

in Table 1 in our previous paper (Klochkova \& Tavolganskava 2010). In this paper, based on the same spectra taken with the 6 -meter telescope, accurate radial velocities of 15 metal-deficient stars 
were published (Klochkova \& Tavolganskava 2010). The list of spectral lines (more than 8000) was made using the VALD database (Piskunov et al. 1995; Kupka et al. 1999). Nearly 860 unblended features from this list were selected for Vr measurements. The standard deviation of the measured velocity does not exceed $\sigma \leq 0.9 \mathrm{~km} / \mathrm{s}$ for stars with the metallicity $[\mathrm{Fe} / \mathrm{H}] \geq-1$, and $\sigma \leq 1.1 \mathrm{~km} / \mathrm{s}$ for stars with $[\mathrm{Fe} / \mathrm{H}] \leq-1$.

\section{Model parameters and elemental abundances determination}

The effective temperature $T_{\text {eff }}$ was determined using the Strömgren $u v b y \beta$ indices and calibration based on the infrared flux method (Alonso 1996). Metallicity values needed for the first iteration of $T_{\text {eff }}$ determination were used from publications, but in the following iterations our spectroscopic $[\mathrm{Fe} / \mathrm{H}]$ values were used. In this procedure from 100 to $230 \mathrm{Fe}$ I lines were taken into account for different stars. Additionally, to control the $T_{\text {eff }}$ values we used the generally adopted spectroscopic way of $T_{\text {eff }}$ determination, forcing independence of $\lg \epsilon(F e)$ on the lower level excitation potential.

Surface gravity was calculated using known relations:

$$
\lg \frac{g}{g_{\odot}}=\lg \frac{\mathcal{M}}{\mathcal{M}_{\odot}}+4 \lg \frac{T_{e f f}}{T_{e f f \odot}}+0.4\left(M_{b o l}-M_{b o l, \odot}\right),
$$

where $M_{b o l}=V+B C+5 \lg \pi+5, \mathcal{M}$ - mass of the star, $M_{b o l}$ is the bolometric luminosity, $V$ is the visual magnitude, $B C$ denotes bolometric correction, $\pi$ is the stellar parallax.

The Hipparcos parallaxes (Perryman et al. 1997) were used for the calculations. Bolometric corrections were calculated using a calibration formula of Flower (1996).

The microturbulent velocity $\xi_{t}$ was determined forcing the independence of the neutral iron abundance on the equivalent width $\mathrm{W}_{\lambda}$ of the line. Atmospheric parameters adopted for 14 stars are presented in Table1, The WIDTH9 code and Kurucz's grid of atmospheric models (Kurucz 1993) were used to calculate chemical abundances. To obtain the normalized logarithmic abundance ratio of elements to iron $[\mathrm{Elem} / \mathrm{Fe}]=\log \left(N_{E l e m} / N_{F e}\right)-\log \left(N_{E l e m} / N_{F e}\right) \odot$ we used solar abundances presented by Asplund et al. (2009). Spectral lines for the calculation were selected using data from VALD (Piskunov et al. 1995; Kupka et al. 1999). The atomic constants of lines needed for abundance calculations were taken from the same database. The list of lines was published earlier by Klochkova et al. (2006) and it is available at http://www.chjaa.org/2006_6_5.html.

The use of échelle spectra observed within a broad short-wavelength spectral range allowed the model atmosphere method to be applied to determine abundances of 20 chemical elements including heavy metals from Sr to Dy presented in Table 3. Abundances of the iron group elements (Cr, Mn, $\mathrm{Co}, \mathrm{Ni}, \mathrm{Zn}$ ), which origin in the equilibrium Si-burning, show very small scattering relative to the Fe abundance. Especially it concerns zinc, whose average relative abundance is $[\mathrm{Zn} / \mathrm{Fe}]=0.06$ for stars with $[\mathrm{Fe} / \mathrm{H}] \leq-1.4$. 


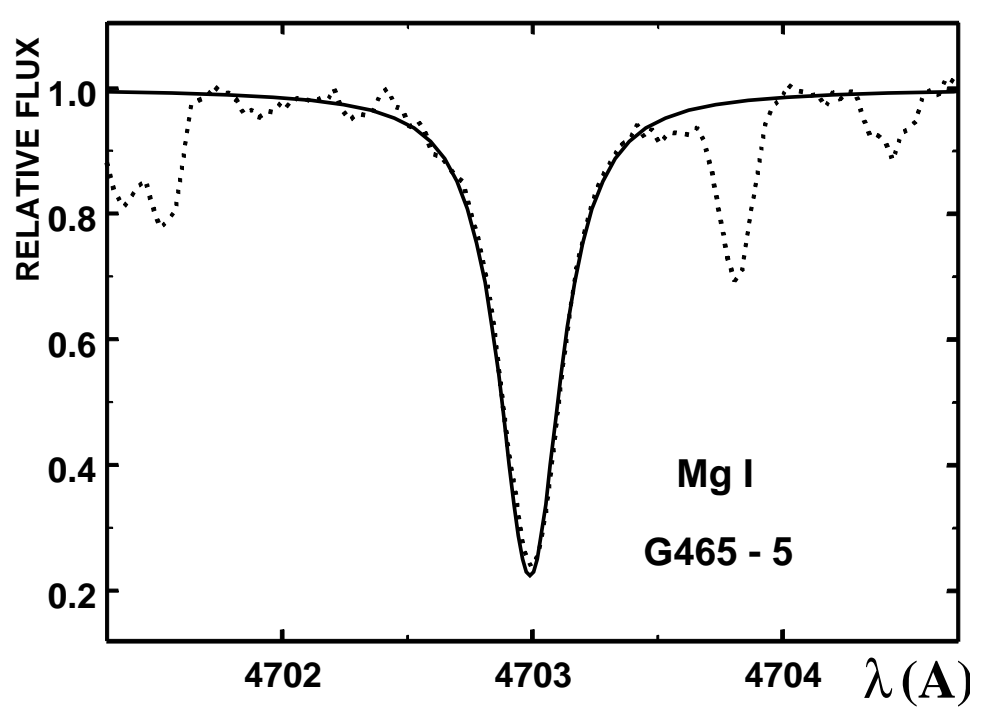

Fig. 1. - The Mg I $\lambda 4703 \AA$ line profile (top) observed in the spectrum of HD 148816 (dotted line). A synthetic profile is shown by a thick line

Abundances of $\mathrm{Mg}, \mathrm{Al}, \mathrm{Sr}$ and $\mathrm{Ba}$ are calculated with a non-LTE approximation (see Table2). The non-LTE abundance elements was determined with a modified MULTI code (Carlsson 1986). Modifications are described by Korotin et al. (1999). Since we use Kurucz's atmosphere models calculated with ATLAS 9 (Kurucz 1993, 2005), the necessary background opacities for MULTI are also from ATLAS 9. In the modified version, the mean intensities that are used to obtain the radiative photoionization rates are calculated for a set of frequencies at each atmospheric layer, and then they are stored in a separate block, where they can be interpolated.

The atomic model of magnesium used in this work is essentially the same as that described by Mishenina et al. (2004). This model consists of 84 levels of Mg I, 12 levels of Mg II and the ground level of Mg III. Within the described system, transitions between first 59 levels of Mg I and ground level of Mg II have been considered. The detailed structure of the multiplets was ignored and each LS multiplet was considered as a single term (more details in Mishenina et al. 2004).

For the non-LTE calculations we adopted an aluminium atomic model that consists of 78 levels of $\mathrm{AlI}$ and 13 levels of AlII. Oscillator strengths and photoionization cross sections were taken from the TOPbase (http://vizier.ustrasbg.fr/topbase/topbase.html). More details see in the paper by Andrievsky et al. (2008).

Since the resonance Sr II and Al I lines in the UV band of the spectrum are somewhat blended with other metallic lines, to compare non-LTE line profiles with the observed spectrum one needs to use a combination of a non-LTE and LTE synthetic spectrum. This was done with the updated code SYNTHV (Tsymbal 1996) which is designed for synthetic spectrum calculations within LTE. With this program we calculated synthetic spectra for selected regions comprising the Sr II and 


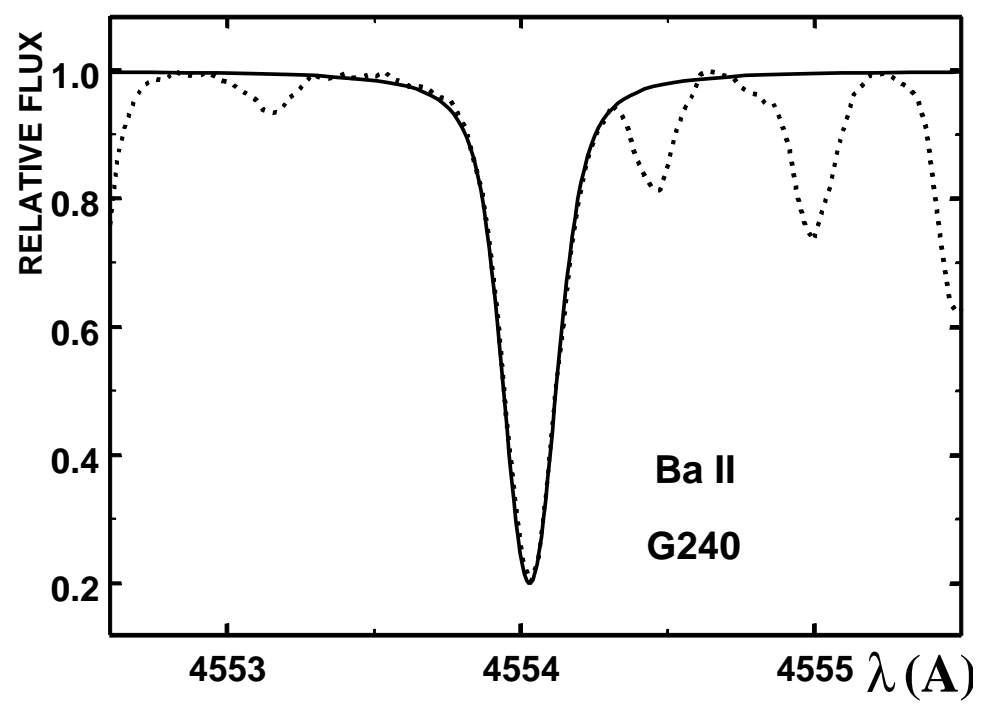

Fig. 2.- The same as in Fig. 1, but for the line BaII $\lambda 4554 \AA$

Al I lines of interest taking into account all the lines from each region listed in the VALD database (Piskunov et al. 1995; Kupka et al. 1999). Then we included corresponding b-factors (factors of deviation from LTE level populations) calculated in MULTI separately for the strontium and aluminium lines into SYNTHV (Tsymbal 1996), where they were used for calculations of the nonLTE line source function.

Our strontium atomic model consists of 44 levels of Sr II with $n<13$ and $l<6$, as well as the ground level of Sr III. Since the ionization potential of the neutral strontium is only $5.7 \mathrm{eV}$, even in atmospheres of cool stars it exists only as of Sr II. Therefore, 24 levels of Sr I were included in the model only to conserve the particle number. Radiative photoionization rates for the $s, p$ and $d$ levels are based on the photoionization cross sections calculated with the quantum defect method (see Andrievsky et al. 2010 for details).

Our barium model contains 31 levels of Ba I, 101 levels of Ba II with $n<50$, and the ground level of Ba III ion. We considered 91 bound-bound transitions in detail. A cause of some uncertainty in the non-LTE analysis of the barium spectrum is the scarce information about the photoionization cross-sections for different levels. We used results obtained using the scaled Thomas-Fermi method (Hofsaess 1979) (for more details see Andrievsky et al. 2009).

To illustrate these calculations, we compare the Mg I $4702 \AA$ profile observed in the HD 148816 spectrum with a theoretical one in Fig. 1. A similar comparison for the lines Ba II $\lambda 4554 \AA$, Eu II $\lambda 4129 \AA$ and Th II $\lambda 4019 \AA$ is presented in Fig. $2-4$.

Aiming to estimate cosmochronological age, the abundance ratio of the radioactive element Th to that of the r-process element Eu is determined for the selected stars (see Table 4). Being independent on the stellar evolution models, the cosmochronological method of the age estimation 
is sensitive both to reliability of stellar nucleosynthesis calculations and to accuracy of heavy metal abundances derived from spectroscopic observations. We determined $\mathrm{Eu}$ and $\mathrm{Th}$ abundances by comparison of the observed and synthetic spectra in the region of the $4129 \AA$ and $4019 \AA$ lines for $\mathrm{Eu}$ and Th, respectively. Synthetic spectra were calculated using a current version of the STARSP package developed by Tsymbal (1996). The Th $4019 \AA$ line is heavily blended (Fe I, Ni I, Co I, V II, $\left.{ }^{13} \mathrm{CH}\right)$, therefore the error of the Th abundance determination is over 0.1 dex. Taking into account these errors in the abundances of $\mathrm{Eu}$ and $\mathrm{Th}$, we (as well as other authors) obtained too large age errors, more than 4 Gyr. For this reason we do not present our age estimations.

\section{Discussion of results}

The stars were related to subpopulations of the Galaxy on the basis of the components of stellar spatial velocities. Then this stratification was refined using elements of Galactic orbits of the stars. To do this, we computed the orbital elements by simulating 30 revolutions of the star around the Galactic center, based on the model of the Galaxy containing a disk, bulge, and extended massive halo (Allen 1991). The Galactocentric distance of the Sun was assumed to be $8.5 \mathrm{kpc}$, the rotational velocity of the Galaxy at the solar Galactocentric distance was $220 \mathrm{~km} / \mathrm{s}$, and the velocity of the Sun with respect to the local standard of rest is $(\mathrm{U}, \mathrm{V}, \mathrm{W})_{\odot}=(-11,14,7.5) \mathrm{km} / \mathrm{s}$ (Ratnatunga et al. 1989). The stars of the thin and thick disc subsystems were separated according to a method that calculates the probability of the star membership in each subsystem using components of their spatial velocity (Koval et al. 2006). Stars of the thick disk and proper halo were separated using total spatial velocity relative to the local standard of rest $-\mathrm{V}_{\mathrm{lsr}}=175 \mathrm{~km} / \mathrm{c}$ (Marsakov, Borkova 2005). The accreted halo stars (i.e. stars of extragalactic origin) were identified using criterion $-\mathrm{V}_{\text {lsr }}>240 \mathrm{~km} / \mathrm{c}$ - these stars have high orbital energies and most of them move on retrograde orbits (Marsakov, Borkova 2006).

In this work, the parameters (including the metallicity) and elemental abundances were determined for 10 stars. We also calculated the $\mathrm{Mg}, \mathrm{Al}, \mathrm{Sr}$, Ba abundances in a non-LTE approximation for 4 stars using parameters that were determined earlier Klochkova et al. (2006). As follows from Table1, the stars have metallicities within a wide interval: $[\mathrm{Fe} / \mathrm{H}]=-0.3 \div-2.9$. The relative abundances [Elem/Fe] presented in Tables 3 and 2 correspond to the expected values according to common behaviour from papers (McWilliam 1997; Roederen 2009). This means that, for example, the $\alpha$-process elements $(\mathrm{Mg}, \mathrm{Si}, \mathrm{Ca}, \mathrm{Ti})$ are overabundant for metal-poor stars at $[\mathrm{Fe} / \mathrm{H}] \leq-1.0$, and the value $[\alpha / \mathrm{Fe}]$ is almost constant with a small dispersion: $[\alpha / \mathrm{Fe}]=+0.3 \div 0.4$. For stars at $[\mathrm{Fe} / \mathrm{H}] \geq-1.0$ abundances of these elements decrease. The star $\mathrm{BD}+80^{\circ} 245$ is an exception.

Our results for $[\mathrm{Mg} / \mathrm{Fe}]$ are very well correspondent to the $[\mathrm{Mg} / \mathrm{Fe}]$-behaviour derived by Gehren et al. (2006) for an extensive stellar sample.

Taking into account kinematical parameters and chemical composition of stars studied, we recognized their belonging to various galactic populations. The most noteworthy result is a peculiar 


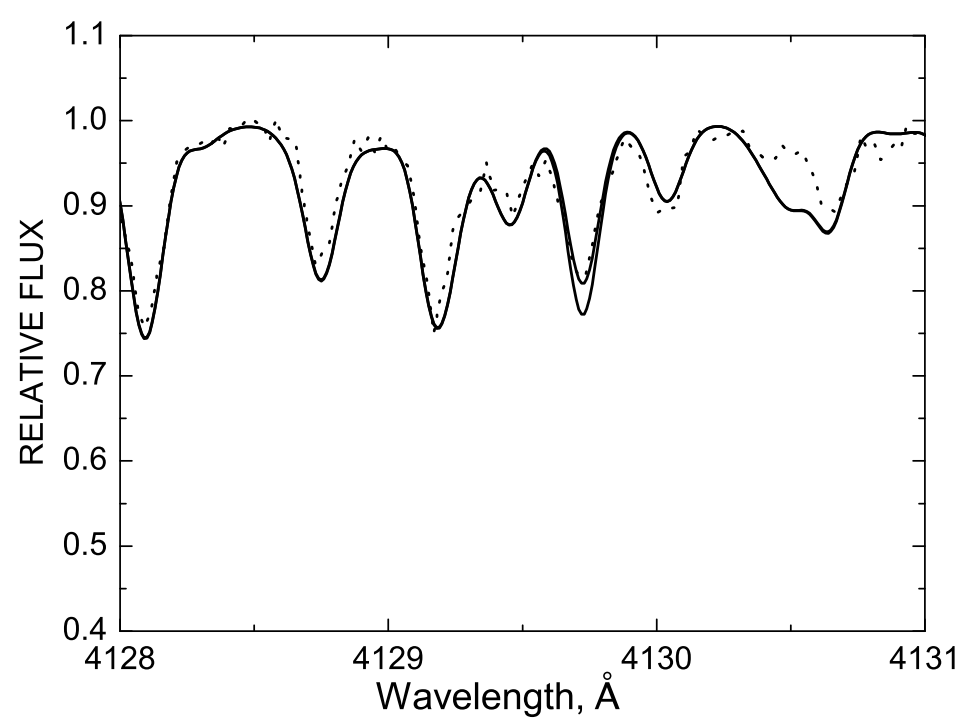

Fig. 3. - The same as in Fig. 1, but for the line Eu II $\lambda 4129 \AA$ in the spectrum of HD 22879

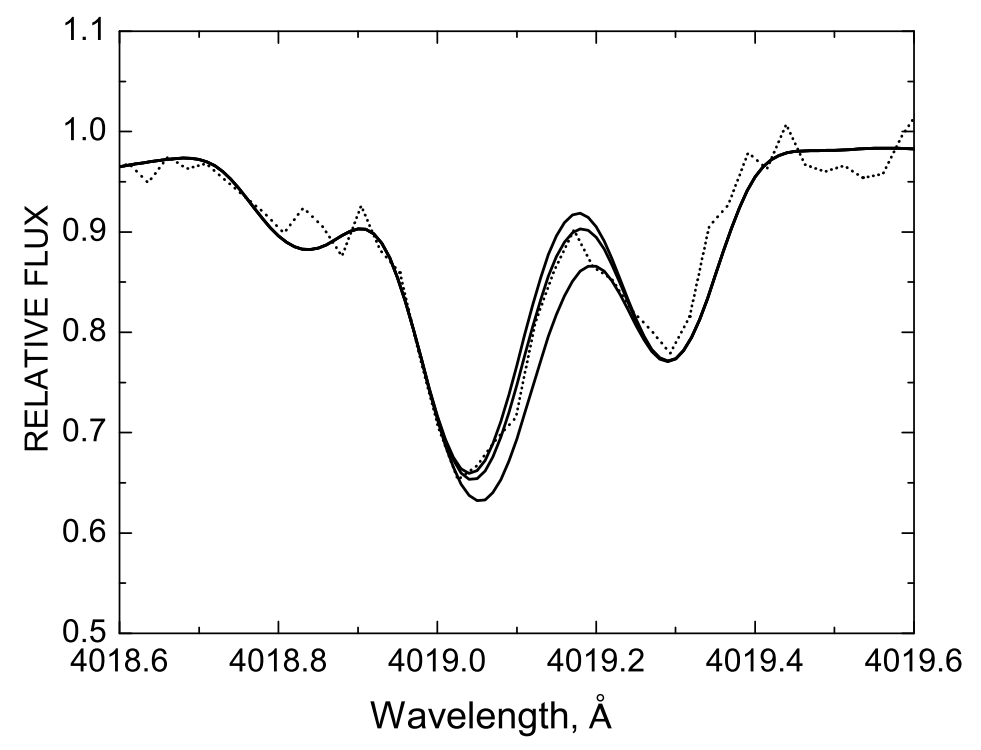

Fig. 4. - The same as in Fig. 1, but for the line Th II $\lambda 4019 \AA$ in the spectrum of HD 5256. Synthetic spectra (from top to bottom) are calculated with $\mathrm{N}(\mathrm{Th})=-12.5,-12.0,-11.5$

chemical abundance pattern in the atmosphere of $\mathrm{BD}+80^{\circ} 245$ whose metallicity is $[\mathrm{Fe} / \mathrm{H}]=-1.71$. The star is allocated with both lowered (for its metallicity) abundances of $\mathrm{Mg}, \mathrm{Al}, \mathrm{Ca}$ and remarkable deficiency of the s-procees element $\mathrm{Ba}:[\mathrm{Ba} / \mathrm{Fe}]=-1.46$. As follows from Table 5 , BD $+80^{\circ} 245$ is also very distant from the Galactic plane $\mathrm{Z}_{\max }=23 \mathrm{kpc}$. Summarizing our results (metallicity, peculiar chemical composition, and kinematical parameters), we classify this star as belonging to the accreted halo. It should be noted that this conclusion is in agreement with earlier results of 
Carney et al. (1997) who revealed similar chemical peculiarities for $\mathrm{BD}+80^{\circ} 245$ and suspected that it is an object originated not in our Galaxy. Now it is known that the galactic halo is not a single homogeneous population, but at least two subpopulations, outer and inner, (or initial and accreted) (Venn et al. 2004; Carollo et al. 2010).

It should be noted that other two stars, $\mathrm{BD}+71^{\circ} 31$ and HD 148816 which kinematically belong to the accreted halo, do not have chemical peculiarities similar to those obtained for $\mathrm{BD}+80^{\circ} 245$.

There is another paradoxical object - a giant HD 115444 with the low metallicity, $[\mathrm{Fe} / \mathrm{H}]=-2.91$. For this star we obtained a peculiar chemical abundance pattern which is in agreement with published earlier by Westin et al. (2000): overdeficiency of Mn and high overabundance of heavy metals from Ba to Dy (Klochkova et al. 2006). We noted especially high excess of the r-process element Europium: $[\mathrm{Eu} / \mathrm{Fe}]=+1.26$. On the one hand, we see a star with low metallicity and large overabundance of heavy metals. In total, the abundances of chemical elements are typical for halo stars. On the other hand, kinematically HD 115444 belongs to the disk population. It is evident that its origin and history do not correspond to each other.

More detailed results concerning chemical abundances, classification by population, and elements of cosmochronology will be presented in the forthcoming paper (Klochkova et al. 2011).

\section{Summary}

Based on spectroscopic data $(\mathrm{R} \geq 60000$ within the wavelength range $3550-5100 \AA)$ taken with the spectrograph NES of the 6-meter telescope, we determined parameters (effective temperature, surface gravity, microturbulent velocity, metallicity, and accurate radial velocity) of a sample of 14 metal-deficient G-K stars with large proper motions. The use of échelle spectra allowed the model atmosphere method to be applied for determination of abundances of 20 chemical elements including heavy metals from $\mathrm{Sr}$ to $\mathrm{Dy}$. Abundances of $\mathrm{Mg}, \mathrm{Al}, \mathrm{Sr}$ and $\mathrm{Ba}$ are calculated within a non-LTE approach.

Taking into account kinematical parameters and chemical composition of the investigated stars, we determined galactic populations they belong to. The chemical composition of the halo star $\mathrm{BD}+80^{\circ} 245$ that is located far from the galactic plane agrees with its belonging to the accreted halo. In addition to the overdeficiency of $\alpha$-process elements, the star has the underabundance of the s-process element barium: $[\mathrm{Ba} / \mathrm{Fe}]=-1.46$. Peculiarities of the chemical composition derived for another metal-deficient star HD 115444 do not correspond to its kinematic parameters. This star also has a non-standard origin.

We conclude that the sample of high proper motion stars we studied is inhomogeneous - it includes objects within a wide metallicity interval $[\mathrm{Fe} / \mathrm{H}]=-0.3 \div-2.9$ that belong to both disk (thin and thick) and halo (inner and accreted). 


\section{Acknowledgements}

V.P. and N.T. are much indebted both to the Russian Foundation for Basic Research for financial support of this work (project 07-02-00247 a) and the Russian Federal program "Observational manifestations of evolution of chemical abundances of stars and Galaxy". T.M. and S.K. are grateful for the support of the Swiss national Science Fund (project SCOPES No. IZ73Z0-128180/1). V.M. is grateful for the financial support of the Ministry of education and science of the Russian Federation (the project P. 685). 


\section{REFERENCES}

Allen, C., Santillan, A. 1991, Rev. Mex. A\&A, 22255

Alonso, A., Arribas, S., Martinez-Roger, C. 1996, A\&A, 313873

Andrievsky, S.M., Spite, M., Korotin, S.A., et al., 2008, A\&A, 481481

Andrievsky, S.M., Spite, M., Korotin, S.A., et al., 2009, A\&A, 4941083

Andrievsky S.M., Spite F., Korotin S.A., et al., 2010, A\&A, 50988

Asplund, M., Grevesse, N., and Sauval, A.J., Scott, P. 2009, ARA\&A, 47481

Carney, B.W., Latham, D.W., Laird, J.B., et al., 1994, AJ, 1072240

Carney, B.W., Wright, J., Sneden, C., et al., 1997, AJ, 114363

Carlsson, M. 1986, Uppsala Obs. Rep., 33

Carollo, D., Beers, T., Chiba, M., et al., 2010, ApJ, 712692

Flower, P. 1996, ApJ, 469355

Gehren, T., J.R. Shi, H.W. Zhang, et al., 2006, A\&A, 4511065

Hofsaess, D. 1979, Atomic Data and Nuclear Data Tables, 24285

Kappelmann, N., Barnstedt, J., Gringel, W., et al. SPIE, 2006, 626625

Klochkova, V.G., Zhao, G., Ermakov, S.V., Panchuk, V.E. Chinese Astron. Astrophys., 2006, 6 579 .

Klochkova, V.G., Tavolganskaya, N.S. Astrophys. Bull., 2010, 6518

Klochkova, V.G., Mishenina, T.V., Panchuk, et al., Astrophys. Bull., 2011, 66 No.1, in press

Korotin, S.A., Andrievsky, S.M., Luck, R.E., 1999, A\&A, 351168

Koval', V.V., Marsakov, V.A., Borkova, T.V. 2006, Astron. Rep., 53785

Kupka, F., Piskunov, N.E., Ryabchikova, T.A., et al., 1999, A\&AS, 138119

Kurucz, R. 1993, ATLAS 9 stellar atmospheres program and 2 kms-1. CD-ROM 13.

Kurucz, R.L. 2005, Memorie della Società Astronomica Italiana Supplementi, 814.

Marsakov, V.A., Borkova, T.V. 2005, Astron. Lett., 31515

Marsakov, V.A., Borkova, T.V. 2006, Astron. Lett., 32604 
McWilliam, A., 1997, ARA\&A, 35503

Mishenina T.V., Soubiran C., Kovtyukh V.V., Korotin S.A. 2004, A\&A, 418551

Panchuk, V., Klochkova, V., Yushkin, M., Najdenov, I. 2009, Journal of Optical Technology, 7687

Perryman, M.A.C., Lindegren, L., Kovalevsky, J., et al., 1997, A\&A, 323 L49

Piskunov, N.E., Kupka, F., Ryabchikova, T.A., et al., 1995, A\&AS, 112525

Ratnatunga, K.U., Bahcall, J. N., and Casertano, S. 1989, ApJ, 339106

Roederer, I. 2009, AJ, 137272

Shustov, B., Sachkov, M., Gomez de Castro, A.I., et al. 2009, Astrophys. Space Sci., 320187

Tsymbal, V.V. 1996, Ap\&SS, 108198

Venn, K.A., Irwin, M., Shetrone, M.D., et al., 2004 AJ, 1281177

Westin, J., Sneden, C., Gustafsson, B., Cowan, J. 2000, ApJ, 530783

Yushkin, M.V., Klochkova, V.G. 2004, Preprint of Special Astrophysical Observatory, No. 2061 
Table 1: Atmospheric parameters and metallicity $[\mathrm{Fe} / \mathrm{H}]$ of program stars. Binary stars are marked in the last column

\begin{tabular}{r|r|r|r|r|l}
\hline HD, BD & $\mathrm{T}_{\text {eff }}$ & $\log \mathrm{g}$ & $\xi_{\mathrm{t}}$ & {$[\mathrm{Fe} / \mathrm{H}]$} & \\
\hline 245 & 5494 & 4.20 & 0.8 & -0.68 & bin \\
$+71^{\circ} 31$ & 6237 & 4.24 & 1.25 & -1.83 & bin \\
5256 & 5212 & 3.80 & 0.5 & -0.39 & \\
$+29^{\circ} 366$ & 5609 & 4.45 & 0.5 & -0.91 & \\
19445 & 5890 & 4.50 & 0.7 & -2.04 & bin? \\
22879 & 5802 & 4.37 & 0.4 & -0.78 & \\
237354 & 5661 & 4.26 & 0.6 & -0.63 & bin \\
$+80^{\circ} 245$ & 5543 & 3.60 & 1.5 & -1.71 & \\
115444 & 4800 & 1.60 & 1.7 & -2.91 & \\
144061 & 5579 & 4.33 & 0.5 & -0.31 & \\
148816 & 5812 & 4.11 & 1.35 & -0.77 & \\
188510 & 5410 & 5.00 & 0.6 & -1.52 & \\
215065 & 5567 & 4.50 & 0.5 & -0.58 & \\
215257 & 5900 & 4.35 & 1.0 & -0.60 & \\
\hline
\end{tabular}


Table 2: Non-LTE abundances of $\mathrm{Mg}, \mathrm{Al}, \mathrm{Sr} \& \mathrm{Ba}$ in the atmospheres of stars studied, $\mathrm{A}(\mathrm{Elem})$. Hydrogen abundance $\mathrm{A}(\mathrm{H})=12.0$

\begin{tabular}{l|r|r|r|r}
\hline \multirow{2}{*}{ Star } & \multicolumn{4}{|c}{$\mathrm{N}(\mathrm{Elem})$} \\
\cline { 2 - 5 } $\mathrm{Mg}$ & $\mathrm{Al}$ & $\mathrm{Sr}$ & $\mathrm{Ba}$ \\
\hline HD 245 & 7.10 & 5.70 & 2.07 & 1.45 \\
HD $+71^{\circ} 31$ & 6.05 & 4.40 & 1.17 & 0.42 \\
BD+29 $59^{\circ} 366$ & 7.20 & 5.70 & 2.32 & 1.60 \\
HD 19445 & 6.09 & 5.85 & 1.92 & 1.14 \\
HD22879 & 7.07 & 5.71 & 1.02 & 0.35 \\
HD 237354 & 7.00 & 6.10 & 2.07 & 1.47 \\
BD+80²45 & 5.80 & 4.60 & 0.47 & 1.40 \\
HD 115444 & 5.44 & 3.45 & 0.06 & -0.06 \\
HD 144061 & 7.20 & 6.00 & 2.42 & 1.60 \\
HD 148816 & 7.13 & 6.15 & 2.07 & 1.27 \\
HD 188510 & 6.33 & 4.03 & 1.03 & 0.71 \\
HD 215065 & 7.20 & 6.10 & 2.17 & 1.40 \\
HD 215257 & 7.14 & 5.23 & 2.27 & 1.74 \\
\hline
\end{tabular}


Table 3: Relative abundances of chemical elements [Elem/Fe]

\begin{tabular}{|c|c|c|c|c|c|c|c|c|c|c|}
\hline \multirow[b]{2}{*}{$\mathrm{HD} / \mathrm{BD}$} & \multicolumn{10}{|c|}{$[$ Elem $/ \mathrm{Fe}]$} \\
\hline & 245 & $+71^{\circ} 31$ & 5256 & $+29^{\circ} 366$ & 22879 & 237354 & $+80^{\circ} 245$ & 144061 & 148816 & 215065 \\
\hline $\mathrm{NaI}$ & 0.10 & & 0.03 & 0.19 & 0.02 & 0.07 & & 0.05 & 0.08 & 0.21 \\
\hline $\mathrm{CaI}$ & 0.09 & 0.31 & 0.02 & 0.14 & 0.10 & 0.12 & -0.24 & -0.07 & 0.15 & 0.11 \\
\hline ScII & 0.10 & 0.23 & 0.34 & 0.23 & 0.17 & 0.28 & -0.31 & 0.25 & 0.06 & 0.21 \\
\hline TiI & 0.09 & 0.33 & 0.08 & 0.13 & 0.23 & 0.21 & -0.19 & 0.03 & 0.20 & 0.20 \\
\hline TiII & 0.34 & 0.33 & 0.25 & 028 & 036 & 0.28 & -0.20 & 0.18 & & 0.25 \\
\hline VI & 0.18 & 0.17 & 0.36 & & 0.24 & 0.20 & -0.22 & 0.26 & 0.17 & 0.32 \\
\hline $\mathrm{CrI}$ & 0.02 & 0.18 & -0.07 & 0.02 & -0.05 & 0.03 & 0.03 & 0.12 & 0.03 & 0.10 \\
\hline CrII & 0.19 & 0.08 & 0.20 & 0.10 & 0.13 & 0.22 & 0.09 & 0.31 & 0.07 & 0.20 \\
\hline MnI & -0.10 & -0.18 & -0.03 & -0.2 & -0.25 & -0.14 & -0.22 & 0.05 & -0.22 & -0.02 \\
\hline FeII & 0.05 & 0.01 & -0.01 & -0.06 & -0.01 & 0.04 & -0.07 & 0.06 & -0.01 & 0.02 \\
\hline $\mathrm{CoI}$ & 0.18 & 0.20 & 0.01 & 0.09 & 0.14 & 0.18 & -0.08 & -0.04 & 0.19 & 0.18 \\
\hline $\mathrm{NiI}$ & 0.08 & 0.15 & 0.09 & 0.02 & 0.00 & 0.09 & -0.05 & 0.14 & 0.03 & 0.11 \\
\hline $\mathrm{ZnI}$ & 0.39 & 0.08 & 0.47 & 0.15 & 0.16 & 0.23 & -0.23 & 0.33 & 0.17 & 0.28 \\
\hline YII & 0.10 & 0.46 & 0.20 & 0.04 & 0.23 & 0.31 & -0.64 & 0.23 & -0.07 & 0.03 \\
\hline $\mathrm{ZrII}$ & 0.09 & 0.30 & 0.22 & 0.04 & 0.21 & 0.25 & 0.24 & 0.24 & 0.04 & 0.02 \\
\hline LaII & 0.28 & 0.44 & 0.30 & 022 & 0.20 & 0.13 & -0.41 & 0.14 & & 0.19 \\
\hline CeII & 0.11 & & 0.03 & 0.08 & -0.06 & 0.04 & & 0.13 & 0.02 & 0.01 \\
\hline NdII & 0.23 & 0.32 & -0.05 & 0.17 & 0.08 & 0.19 & & 0.22 & 0.19 & -0.03 \\
\hline SmII & 0.26 & & 0.15 & & 0.25 & 0.28 & & 0.06 & 0.10 & 0.19 \\
\hline GdII & 0.41 & & 0.09 & 0.36 & 0.07 & 0.46 & & 0.01 & 0.11 & 0.26 \\
\hline DyII & 0.46 & & 0.46 & 0.34 & 0.34 & 0.09 & & 0.23 & 0.06 & 0.26 \\
\hline
\end{tabular}


Table 4: Abundances $\log \epsilon(\mathrm{Eu})$ and $\log \epsilon(\mathrm{Th})$, relative abundances $[\mathrm{Eu} / \mathrm{H}]$ and $[\mathrm{Th} / \mathrm{H}]$, and ratios $[\mathrm{Eu} / \mathrm{Fe}]$ and $[\mathrm{Th} / \mathrm{Fe}]$. Solar abundances $\log \epsilon(\mathrm{Eu})=0.51$ and $\epsilon(\mathrm{Th})=0.02$ are taken from (Asplund et al. 2009)

\begin{tabular}{r|r|r|r|r|r|r|r|r|r}
\hline $\mathrm{HD} / \mathrm{BD}$ & 245 & 5256 & $+29^{\circ} 366$ & 22879 & 237354 & 115441 & 144061 & 148816 & 215065 \\
\hline $\log \epsilon(\mathrm{Eu})$ & 0.30 & 0.40 & 0.00 & 0.20 & 0.30 & -1.14 & 0.40 & 0.20 & 0.30 \\
{$[\mathrm{Eu} / \mathrm{H}]$} & -0.21 & -0.11 & -0.51 & -0.31 & -0.21 & -1.65 & -0.11 & -0.31 & -0.21 \\
{$[\mathrm{Eu} / \mathrm{Fe}]$} & 0.47 & 0.28 & 0.40 & 0.47 & 0.42 & 1.26 & 0.20 & 0.46 & 0.37 \\
\hline $\log \epsilon(\mathrm{Th})$ & -0.20 & 0.00 & & -0.20 & 0.00 & -1.62 & 0.00 & & \\
{$[\mathrm{Th} / \mathrm{H}]$} & -0.22 & -0.02 & & -0.22 & -0.02 & -1.64 & -0.02 & & \\
{$[\mathrm{Th} / \mathrm{Fe}]$} & 0.46 & 0.36 & & 0.56 & 0.61 & 1.27 & 0.29 & & \\
$\mathrm{Th} / \mathrm{Eu}$ & -0.50 & -0.40 & & -0.40 & -0.30 & -0.48 & -0.40 & & \\
\hline
\end{tabular}


Table 5: Distance d, spatial velocity components $\mathrm{U}, \mathrm{V}, \mathrm{W}, \mathrm{V}_{\mathrm{lsr}}$ and parameters of the stellar orbits calculated: e - orbital eccentricity, $\mathrm{Z}_{\max }$ - maximum distance from the Galactic plane, $\mathrm{R}_{\max }-$ apogalactic radius. Galactic subsystems to which the stars belong is noted in the last column: 1 - thin disk, 2 - thick disk, 3 - initial halo, 4 - accreted halo, 1-2 - the star rather belongs to the thin disk than to the thick one; 3-2 - the star rather belongs to the initial halo than to thick disk

\begin{tabular}{r|c|r|r|r|r|r|r|r|r}
\hline $\mathrm{HD} / \mathrm{BD}$ & $\mathrm{d} \pm \sigma$ & $\mathrm{U}$ & $\mathrm{V}$ & $\mathrm{W}$ & $\mathrm{V}_{\mathrm{lsr}}$ & $\mathrm{e}$ & $\begin{array}{r}\mathrm{Z}_{\max } \\
\mathrm{kpc}\end{array}$ & $\begin{array}{r}\mathrm{R}_{\max } \\
\mathrm{kpc}\end{array}$ \\
\hline 245 & $61.58 \pm 2.7$ & -47 & -104 & -46 & 108 & 0.44 & 0.6 & 9.0 & 2 \\
$+71^{\circ} 31$ & $165.56 \pm 32.4$ & -171 & -231 & 11 & 274 & 0.99 & 8.8 & 12.1 & 4 \\
5256 & $89.37 \pm 6.7$ & -103 & -77 & 44 & 125 & 0.44 & 1.1 & 10.9 & 2 \\
$+29^{\circ} 366$ & $56.63 \pm 5.5$ & -63 & -72 & -52 & 94 & 0.34 & 0.8 & 9.6 & 2 \\
19445 & $38.68 \pm 1.7$ & 153 & -120 & -71 & 207 & 0.61 & 1.5 & 11.3 & $3-2$ \\
22879 & $24.35 \pm 0.5$ & -110 & -74 & -66 & 133 & 0.44 & 1.3 & 11.2 & 2 \\
237354 & $83.96 \pm 11.4$ & 32 & -138 & -123 & 179 & 0.49 & 4.0 & 8.7 & $3-2$ \\
$+80^{\circ} 245$ & $255.75 \pm 88.4$ & -199 & -367 & 226 & 465 & 0.69 & 23.0 & 28.9 & 4 \\
115444 & $281.69 \pm 97.0$ & 54 & -64 & -12 & 84 & 0.26 & 0.3 & 8.9 & $1-2$ \\
144061 & $29.11 \pm 1.0$ & -31 & -6 & -21 & 26 & 0.14 & 0.2 & 10.3 & 1 \\
148816 & $41.08 \pm 1.5$ & 80 & -263 & -87 & 280 & 0.90 & 5.2 & 9.4 & 4 \\
188510 & $39.49 \pm 1.8$ & -167 & -105 & 31 & 187 & 0.64 & 0.8 & 13.2 & $3-2$ \\
215065 & $29.37 \pm 0.5$ & -35 & -64 & 12 & 62 & 0.27 & 0.3 & 9.0 & 1 \\
215257 & $42.27 \pm 1.7$ & -68 & 19 & 37 & 78 & 0.30 & 1.0 & 14.0 & 2 \\
\hline
\end{tabular}

